\title{
Clinical utility of cerebrospinal fluid-derived circular RNAs in lung adenocarcinoma patients with brain metastases
}

\author{
Zhen Wang ${ }^{1 *}\left(\mathbb{D}\right.$, Ruoying $\mathrm{Yu}^{2}$, Xiaoxi Chen ${ }^{2}$, Hua Bao ${ }^{2}$, Ran $\mathrm{CaO}^{2}$, An-Na Li ${ }^{1}$, Qiuxiang Ou², Hai-Yan Tu', \\ Qing Zhou', Xue Wu' ${ }^{2}$, Zhi-Bo Lin ${ }^{1,3}$ and Yi-Long Wu'
}

\begin{abstract}
Background: Free circular RNAs(circRNAs) escaping from primary lesion of cancer to brain are strictly regulated by blood-brain barrier and therefore cerebrospinal fluid (CSF) circRNAs have potential advantage in exploring biomarkers and mechanism of brain metastasis in lung cancer.

Methods: We collected paired cerebrospinal fluid, plasma and tumor tissues from 21 lung adenocarcinoma (ADC) patients with brain metastases (BM) and performed RNA sequencing.

Results: Compared to tumor tissue and plasma, circRNAs in CSF were characterized by lower number of spieces but higher abundance. Notably, CSF-circRNAs displayed high heterogeneity among different BM lung ADC patients. A total of 60 CSF-circRNAs was identified and associated with shorten overall survival. The circRNA-miRNA-mRNA network analysis revealed that the 60 CSF-circRNAs involved in cancer-associated pathways, and five of them showed strong association with WNT signaling pathway. Validation by RT-PCR of CSF and in vitro experiments of the five candidate circRNAs support their potential roles in cell proliferation and invasion.

Conclusions: In summary, our results depicted the heterogenous CSF-circRNAs profiles among BM lung ADC and implied that CSF-circRNAs may be promising prognosis-related biomarkers.
\end{abstract}

Keywords: Circular RNA, Cerebrospinal fluid, Lung ADC brain metastasis, Overall survival

\section{Introduction}

Brain metastasis represents a great challenge among patients with lung cancer [1]. About half of brain metastasis in solid tumors were due to lung cancer $[2,3]$. More than $40 \%$ of lung cancer develop brain metastases (BM) and the risk keeps increasing during their course of disease [1] and BM is usually associated with poor outcomes $[1,3,4]$.

\footnotetext{
*Correspondence: wangzhen@gdph.org.cn

${ }^{1}$ Guangdong Provincial People's Hospital, Guangdong Academy

of Medical Sciences, Guangdong Lung Cancer Institute, Zhong Shan 2nd road 106, Guangzhou 510080, Guangdong, China

Full list of author information is available at the end of the article
}

The difficulties in obtaining tumor tissue samples from BM largely preclude the molecular characterization and monitoring of disease [5]. Liquid biopsies exhibit advantages in molecularly characterizing brain metastatic cancers, however, circulating tumor DNA (ctDNA) in plasma have obvious limitations, e.g. limited detectable amount $[6,7]$. Frequently renewed cerebrospinal fluid (CSF) keeps close contact with brain malignancies $[8,9]$ therefore provides a better solution as reservoir of tumor-related/secreted molecules $[5,10]$. On this basis, simultaneously profiling the molecular characteristics of CSF, tumor tissues and plasma would be of great help in exploring the pathogenesis of brain metastasis in lung cancer.

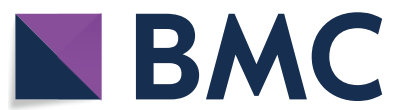

(c) The Author(s) 2022. Open Access This article is licensed under a Creative Commons Attribution 4.0 International License, which permits use, sharing, adaptation, distribution and reproduction in any medium or format, as long as you give appropriate credit to the original author(s) and the source, provide a link to the Creative Commons licence, and indicate if changes were made. The images or other third party material in this article are included in the article's Creative Commons licence, unless indicated otherwise in a credit line to the material. If material is not included in the article's Creative Commons licence and your intended use is not permitted by statutory regulation or exceeds the permitted use, you will need to obtain permission directly from the copyright holder. To view a copy of this licence, visit http://creativecommons.org/licenses/by/4.0/. The Creative Commons Public Domain Dedication waiver (http://creativeco mmons.org/publicdomain/zero/1.0/) applies to the data made available in this article, unless otherwise stated in a credit line to the data. 
Different from DNA biomarkers, RNA can provide dynamic insights into the tumor states and regulatory processes [11]. Circular RNAs (circRNAs) generated by back-splicing involve in tumor pathogenesis through mechanisms such as microRNA (miRNA) sponges, combining RNA binding proteins etc. to regulate transcription and translation [12] in a wide variety of cancers including hepatocarcinoma [13], lung [14], gastric [15], colorectal [16], breast [17], and other cancers [18]. Some specific circRNAs present as promising biomarkers or therapeutic targets [18].

Free circRNAs escaping from primary solid tumors (e.g. lung cancer) to the brain is strictly regulated by presence of blood-brain barrier [19]. CSF-circRNAs could facilitate investigation due to their high abundance and conservation in the brain $[20,21]$. CircRNAs commonly shared between primary and brain metastatic tumors are likely to account for some mechanism underlying lung cancer BM as well as be potential biomarkers.

Most studies specified circRNAs in certain type of tissues [13-17, 22-25] and seldom compared circRNAs across samples (e.g. cerebrospinal fluids, plasma, tumor tissues) [26]. In this study, we profiled circRNAs in 21 lung cancer patients with brain metastases by comparing paired samples including cerebrospinal fluid, plasma, and tumor tissue. A total of 60 candidates lung cancerassociated CSF-circRNAs were identified as close correlation with clinical outcomes. Bioinformatics analysis and preliminary validation concentrated on five circRNAs in the top-ranked Wnt signaling pathway supporting their possible roles in cell proliferation and invasion. Our data revealed CSF-circRNAs might be promising prognosisrelated biomarkers in the lung ADC patients with brain metastases.

\section{Materials and methods Patient sample collection}

Patients diagnosed with lung adenocarcinoma by histology and BM by magnetic resonance imaging (MRI) were recruited. Peripheral blood, tumor tissue and CSF were collected for pathological and genetic analysis. The study was approved by local independent ethics committees and complied with the Declaration of Helsinki and Good Clinical Practice principles and written informed consent forms were obtained.

\section{Targeted generation sequencing and data processing}

DNA extraction, library preparation and targeted enrichment were carried out as previously described $[27,28]$. In brief, genomic DNA from whole blood were extracted using the DNeasy Blood \& Tissue Kit (Qiagen) according to the manufacturer's protocols. FFPE samples were de-paraffinized with xylene followed by genomic DNA extraction using QIAamp DNA FFPE Tissue Kit (Qiagen) following the manufacturer's instruction. CSF was obtained through lumbar puncture as a standard practice for lung cancer patients with brain metastasis following National Comprehensive Cancer Network (NCCN) guidelines. Within $4 \mathrm{~h}$ of collection, the cellular fraction was removed by two-step centrifugation at $4{ }^{\circ} \mathrm{C}$ including $1900 \mathrm{~g}$ for $10 \mathrm{~min}$ then $16,000 \mathrm{~g}$ for $10 \mathrm{~min}$. Plasma samples were first separated with blood cell sediment after centrifuging whole blood samples at $4{ }^{\circ} \mathrm{C}$ for $10 \mathrm{~min}$ $1600 \mathrm{~g}$, then recentrifuged at $4{ }^{\circ} \mathrm{C} 16,000 \mathrm{~g}$ for $10 \mathrm{~min}$. Both cfDNA from CSF and plasma were extracted using Qiagen QIAmp Circulating Nucleic Acid Kit (Qiagen, Germany) following the manufacturer's protocols. Genomic DNA from tumor tissue samples was extracted using QIAmp DNA FFPE Tissue Kit (Qiagen, Germany) following the manufacturer's protocols. Plasma sample was first centrifuged at high speed to remove any cell debris, followed by cfDNA extraction from the supernatant using QIAamp Circulating Nucleic Acid Kit (Qiagen). Sequencing libraries were prepared using the KAPA Hyper Prep Kit (KAPA Biosystems) according to manufacturer's suggestions for different sample types. In brief, cfDNA or fragmented genomic DNA underwent endrepairing, A-tailing and ligation with indexed adapters sequentially, followed by size selection using Agencourt AMPure XP beads (Beckman Coulter). Hybridizationbased target enrichment was carried out with pan-cancer gene panel of 139 cancer-relevant genes, and xGen Lockdown Hybridization and Wash Reagents Kit (Integrated DNA Technologies). Captured libraries by Dynabeads M-270 (Life Technologies) were amplified in KAPA HiFi HotStart ReadyMix (KAPA Biosystems) and quantified by qPCR using the KAPA Library Quantification Kit (KAPA Biosystems) for sequencing.

The libraries were paired-end sequenced on NGS platforms (Illumina) according to the manufacturer's instructions. Trimmomatic [29] was used for FASTQ file quality control (below 15 or $\mathrm{N}$ bases were removed). Reads were then mapped to the reference Human Genome (hg19) using Burrows-Wheeler Aligner (BWA-mem, v0.7.12; https://github.com/lh3/bwa/tree/master/bwakit). Local realignment around the indels and base quality score recalibration was applied with the Genome Analysis Toolkit (GATK 3.4.0; https://software.broadinstitute. org/gatk/), which was also applied to detect germline mutations. VarScan2 [30] was used for somatic mutation detection. Somatic variant calls with at least $0.2 \%$ mutant allele frequency (MAF) and with at least three supporting-reads from both directions were retained. Common SNPs were filtered out using dbSNP (v137) and the 1000 Genomes database, followed by annotation using ANNOVAR [31]. Genomic fusions were identified by 
FACTERA [32] with default parameters. Copy-number variations (CNVs) were detected using ADTEx (http:// adtex.sourceforge.net) with default parameters. Somatic CNVs were identified using paired normal/tumor samples for each exon with the cut-off of 0.65 for copy-number loss and 1.50 for copy-number gain.

\section{RNA-sequencing}

The RNA-sequencing was performed following previous report [33]. Tumor tissue total RNA was extracted with miRNeasy mini-Kit (Qiagen, Hilden, Germany) following instructions, which concentration was assessed using NanoDrop-100 (Thermo Fisher Scientific, Wilmington, DE, USA) and Qubit RNA HS Assay Kit with Qubit 3.0 fluorometer (Life Technologies). Quality control (QC) process was completed via the Agilent 2100 bioanalyzer. Sequencing libraries were constructed with $1000 \mathrm{ng}$ total RNA using KAPA RNA HyperPrep Kit and RiboErase (KAPA Biosystems, Wilmington, MA). cDNA was synthesized after ribosomal RNA depletion and fragmentation for $6 \mathrm{~min}$ at $94^{\circ} \mathrm{C}$. Prior to sequencing on HiSeq4000 platform, libraries were examined for quality and quantity with KAPA Library Quantification Kit (KAPA Biosystems) using qRT-PCR (CFX384 real time system, Bio-Rad Laboratories).

Plasma and CSF samples stored at $-80{ }^{\circ} \mathrm{C}$ were centrifuged at $16,000 \mathrm{~g}$ for $10 \mathrm{~min}$. The supernatants were transferred to new tubes for RNA extraction. Total RNA was isolated using Norgen Plasma/Serum Circulating and Exosomal RNA Purification Mini Kit (NorgenBiotek, ON, Canada), which concentration was determined using NanoDrop-100 (Thermo Fisher Scientific, Wilmington, DE, USA) and Qubit microRNA Assay Kit. Sequencing libraries were generated using SMARTer Stranded Total RNA-Seq Kit v2-Pico Input Mammalian (Pico v2, total RNA, Takara). Sequencing was performed on Illumina Hiseq 4000 machine.

\section{Data processing}

The RNA-Seq reads passing queue thresholds were trimmed of adaptor sequences and aligned to the GRCh37 reference genome with Gencode (v24lift37) [33] annotation using STAR (v2.5.3) [33]. RNA expression abundance was quantified with raw counts from STAR by setting quantMode for GeneCounts parameter. Trimmed Means of M-Values (TMM) normalization was performed on the library size adjusted read counts, which was further converted to FPKM with edgeR (v3.16.5) [33]. Total exon length was calculated using the GenomicFeatures package [34] and used in the FPKM calculation. The number of circRNA species was defined as the number of circRNAs with read counts $>0$. The QC information of samples was shown in Additional file 1: Table S1.
The median number of circRNA reads was 4024, which was similar to the reported range from other studies [35, 36]. The median percentage of total reads mapping was $89.34 \%$. The median percentage of high-quality (HQ) reads were $97.90 \%$. The median of $\mathrm{HQ} \% \mathrm{Q} 30$ and raw reads \%Q30 were 94.03 and 90.64 respectively, suggesting the reliability of the next-generation sequencing results.

\section{Circular RNA identification}

The back-splicing reads were extracted from STAR results (chimeric.out.junction) and annotated using CIRCexplorer (v2.3.0) [33]. The identified CircRNAs for each sample were combined together and back-splicing reads numbers were extracted for the abundance prediction. Consistent with previous report [33], the effective length for circRNA was twice the read length less twice the anchor-size since only reads on this range could be unbiased evidence supporting circRNA. On this basis, FPKM was calculated using edgeR(v3.16.5) [33] and normalized with library sizes and normalization factors from linear transcripts for the same sample. Multiple circRNAs generated from the same gene were treated separately. Principal component analysis (PCA) was performed with the FPKMs of circRNAs (Additional file 2: Table S2) from different sample types. The samples from different batches (a, b, c, d, e, f) are clustered together and not clustered according to the batches, suggesting that the batch effect was limited (Additional file 6: Figure S1).

\section{Survival analysis}

Cox proportional hazards model in $\mathrm{R}$ Survival package (v2.44-1.1) was used to identify independent predictors of survival either without (univariate) or with (multivariate) adjustment for relevant clinical covariates (age, gender, smoking history, metastasis). Patient groups were dichotomized by the expression of each circRNA or the median number of detected circRNA species in the 60 candidates list (circRNA-low: less than median, circRNA-high: more than median). The Kaplan-Meier survival curves were constructed using R Survminer package (v0.4-4) and the log-rank test was used to compare survival time between groups.

\section{Construction of circRNA-miRNA-mRNA network}

One of the first and most investigated functions of circRNAs is that of a miRNA sponge that circRNAs binds to miRNAs and consequently represses their function [37]. Since circRNA are created by back-splicing events from linear mRNA and the miRNA sponge process is similar to miRNA-mRNA interaction, so we used miRanda software [38], a miRNA target scanner that aims to predict mRNA targets for microRNAs, to predict the interacted 
circRNA-miRNA interaction. First, miRNA-binding sites in circRNAs were extracted from the $5^{\prime}$ and $3^{\prime}$ ends (30 nt) and reverse-joined to produce a backsplice junction. Then the list of miRNAs that potentially bind to the given circRNA is generated by miRanda software using mature miRNA sequences from miRBase. To reduce the number of predicted miRNA binding sites, the parameter '-strict' is applied when using miRanda [39]. The threshold miRanda-type score was set as greater than 170 , and energy was set as less than -30 with other default parameters. Finally, a total of 48 circRNAs and associated mRNAs and miRNAs were included. Then the miRNA-mRNA interaction was delineated with miRwalk [40] with default parameters. The graph of the circRNA-miRNA-mRNA network was drawn with the help of Cytoscape 3.7.1 [41]. The circRNAs, miRNAs and mRNAs of the circRNA-miRNA-mRNA network were shown in Additional file 3: Table S3. For the 5 annotated with the top-rank Wnt signaling pathway, Wnt pathwayrelated miRNAs and corresponding target genes were selected for each circRNA and used to generate the graphic interacting network.

\section{Co-expression of circRNA-mRNA and gene enriched pathways}

To determine the association between the expression of circRNA and the regulation of mRNA co-expression, the Pearson correlation coefficient (PCC) between 60 selected circRNA and mRNA were compared. The absolute value of parameter $P C C \geq 0.70$ and $P$ value $<0.01$ was accepted and reserved for pathway analysis. A total of 52 circRNAs were included. The co-expressed mRNAs were shown in Additional file 4: Table S4.

\section{Annotation of circRNA co-expressed and target genes enriched pathways}

GO and KEGG analysis of the co-expressed genes corresponding to the 60 circRNAs. The corresponding gene numbers were counted for Gene Ontology (GO) and Encyclopedia of Genes and Genomes (KEGG) pathway analysis, and significant correlations between target genes and their associated functions and pathways were assessed based on a threshold of $\mathrm{P}<0.05$. The function of target genes of 60 circRNAs based on circRNAmiRNA-mRNA network were annotated using Database for Annotation, Visualization and Integrated Discovery (DAVID) [42]. For clarity, functional annotations for target genes were focused on pathways in cancer. Finally, top-ranked signaling pathways and biological processes were selected on the basis of either gene numbers or p-values in each cluster.

\section{Lentiviral infection and cell proliferation}

Lentiviruses used for circRNAs knockdown and the corresponding negative controls were purchased from Genechem. Short hairpin RNA (shRNA) targeting NOB1 gene was used as positive control (shPC) and the target sequence was AGGAGGAGGAGG AGGAAGAAA. The lentiviral vectors were separately transfected into A549 and HCC827 cells. Infection efficiency after $48-72 \mathrm{~h}$ was determined by GFP expression observed under a fluorescence microscope. Then the cells were harvested for cell proliferation analysis on a daily basis from day 1 to day 5 using Celigo Cell Cytometer (Nexcelom, USA). All experiments were performed in duplicates and repeated at least three times independently.

\section{Real-time PCR quantification}

Total RNA was extracted using the SuperfecTRI, Total RNA Isolation Reagent (Trizol, Pufei, China). One $\mu \mathrm{g}$ total RNA was used for cDNA synthesis with M-MLV reverse transcriptase (Promega, USA). RT-qPCR was performed using SYBR Master Mixture (TAKARA, China). Primers were purchased from Ribobio (http:// www.ribobio.com/). Results were normalized against GAPDH expression.

\section{MTT cell proliferation assay}

The proliferation activity of cells was measured by an MTT assay. The cells (average $2 \times 10^{3}$ cell/well) were seeded into 96-well plates with DMEM medium containing $10 \%$ FBS. MTT solution $(5 \mathrm{mg} / \mathrm{mL}, 20 \mu \mathrm{L})$ was added to each well and the plates were incubated at $37^{\circ} \mathrm{C}$ for $4 \mathrm{~h}$ and the supernatant was removed. After dissolving the formazan crystals in $100 \mu \mathrm{L}$ of DMSO for five minutes, the absorbance of each well was measured using a spectrophotometer (Thermo Fisher Scientific, Vantaa, Finland) at 490/570 $\mathrm{nm}$.

\section{Invasion assays}

For invasion assays, A549 cells transfected with different shRNAs were seeded into the upper chamber of the Transwells (Corning, sigma-aldrich, USA) according to the manufacturer's instructions. Medium containing $10 \%$ FBS was added to the lower chamber for $24 \mathrm{~h}$. Cotton swabs were used to remove the non-invading cells that remained in the top chamber, and the cells that had invaded to the underside of the membrane were fixed in $4 \%$ paraformaldehyde for $30 \mathrm{~min}$ and stained 
with $0.5 \%$ crystal violet. Finally, images were obtained under a light microscope at 100X lens and 200X lens.

\section{Colony formation assay}

Colony formation was performed as described previously [43]. A total of $4 \sim 10 \times 10^{2}$ shRNA-transfected A549 cells suspended in DMEM medium containing $10 \%$ fetal bovine serum were plated in 6-well plates. Each well was done in triplicate. The plates were incubated at $37{ }^{\circ} \mathrm{C}$ in a $5 \% \mathrm{CO} 2$ incubator for 14 days or until colonies with more than 50 cells. The cells were then fixed with $4 \%$ polyoxymethylene and stained with crystal violet. The experiments were done at least three times.

\section{RT-PCR and NGS validation}

The primers for RT-PCR were designed according to the sequences of circRNA junction sites. Cell-free RNAs from CSF were extracted using Norgen Plasma/ Serum Circulating and Exosomal RNA Purification Mini Kit (Slurry Format) according to the manufacturer's instructions. cDNAs were synthesized using the SuperScript VILO Master Mix and used as templates for PCR amplification using Invitrogen ${ }^{\mathrm{TM}}$ Platinum $^{\mathrm{TM}}$ SuperFi ${ }^{\mathrm{TM}}$ PCR Master Mix. Then the PCR products were subjected to agarose gel electrophoresis and PCR product libraries were constructed with cDNA segments extracted from gel using KAPA Hyper Prep C Kit according to the manufacturer's instructions. Lastly, NOVAseq NGS was performed and the sequencing results were compared to the sequence of each circRNA junction site respectively.

\section{Statistical analysis}

Statistical analyses were performed with the $\mathrm{R}$ package (v3.4.4). The Wilcoxon signed-rank test was used to analyze differences regarding the expression level of circRNAs and mRNAs. Pearson's Coefficient was applied to compare the circRNA data and clinical results. $P<0.05$ was set to be statistically significant.

Overall survival was defined from the diagnosis of brain metastases to death from any cause or last followup. Median follow up time was calculated using reverse Kaplan-Meier, which was obtained by reversing the event indicator so that the outcome became being censored. The Kaplan-Meier survival curves were constructed using R Survminer package (v0.4-4) and the log-rank test was used to compare survival time between groups.

Cox proportional hazards model in $\mathrm{R}$ Survival package (v2.44-1.1) was established to identify independent predictors of survival either without (univariate) or with (multivariate) adjustment for relevant clinical covariates (age, gender, smoking history, metastasis).

Patient groups were dichotomized by the expression of each circRNA or the median number of detected circRNA species in the 60 candidates list (circRNA-low: less than median, circRNA-high: more than median).

Table 1 Patient demographic and clinical characteristics

\begin{tabular}{|c|c|}
\hline Character & $\begin{array}{l}\mathrm{N}(\%) \\
\text { Total } \mathrm{N}=21\end{array}$ \\
\hline \multicolumn{2}{|l|}{ Age at baseline, years } \\
\hline Median & 55 \\
\hline Range & $39-74$ \\
\hline \multicolumn{2}{|l|}{ Sex } \\
\hline Male & $9(43 \%)$ \\
\hline \multicolumn{2}{|l|}{ Smoking status } \\
\hline Current smoker & $1(5 \%)$ \\
\hline Former smoker & $5(24 \%)$ \\
\hline Never smoker & $15(71 \%)$ \\
\hline \multicolumn{2}{|l|}{ Activating mutation } \\
\hline EGFR mut & $17(81 \%)$ \\
\hline$A L K$ rearrangement & $3(14 \%)$ \\
\hline KRAS mut & $1(5 \%)$ \\
\hline \multicolumn{2}{|l|}{ LMC } \\
\hline Yes* $^{*}$ & $5(24 \%)$ \\
\hline \multicolumn{2}{|l|}{ Baseline PS } \\
\hline 0 & $1(5 \%)$ \\
\hline 1 & $19(90 \%)$ \\
\hline 2 & $1(5 \%)$ \\
\hline \multicolumn{2}{|l|}{ Treatment received } \\
\hline$\left.\mathrm{TK}\right|^{* *}$ & $9(43 \%)$ \\
\hline TKI + SRS of BM & $3(14 \%)$ \\
\hline $\mathrm{TKI}+$ resection of $\mathrm{BM}$ & $1(5 \%)$ \\
\hline $\mathrm{TKI}+\mathrm{bev}$ & $1(5 \%)$ \\
\hline Chemotherapy & $3(14 \%)$ \\
\hline $\mathrm{Pem}+\mathrm{CBP}+\mathrm{Bev}$ & $1(5 \%)$ \\
\hline Pem + Bev & $1(5 \%)$ \\
\hline Paclitaxel + CBP + Bev & $1(5 \%)$ \\
\hline WBI + BSC & $2(10 \%)$ \\
\hline BSC & $2(10 \%)$ \\
\hline
\end{tabular}

EGFR epidermal growth factor receptor, ALK anaplastic lymphoma kinase, KRAS Kirsten rat sarcoma viral oncogene homologue, mut mutant, $B M$ brain metastasis, $L M C$ leptomeningeal carcinomatosis, PS performance status, TKI tyrosine kinase inhibitor, SRS stereotactic radiosurgery, Pem pemetrexed, $C B P$ carboplatin, Bev bevacizumab, WBI whole brain irradiation, $B S C$ best support care

* Patient was considered to have LMC should had at least one positive result of cerebrospinal fluid (CSF) cytology and brain magnetic resonance imaging (MRI). Three patients were diagnosed with LMC by CSF cytology, brain MRI, accompanied by neurological symptoms, which included headache, confusion, cognitive impairment and psychiatric disorders. The other two patients were diagnosed with LMC by brain MRI without symptom or CSF cytology

** Details of TKI regimens are shown in Additional file 5: Table S5 


\section{Results}

Between May 14, 2018 and October 26, 2015, a total of 21 lung adenocarcinoma (ADC) patients with brain metastases were selected. Patients' clinicopathological data including sex, smoking status, specimen type, genomic alterations (EGF, ERBB2, ALK, KRAS, TP53) and treatment regimens were summarized (Table 1 and Additional file 5: Table S5). The median age was 55 years old, ranging from 39 to 74 . There were 12 (57\%) female and $9(43 \%)$ male patients. Around $71 \%$ of patients were non-smokers while the rest (29\%) were smokers. The size and number of BM lesions were available in 20 patients. Combining CSF cytology, brain MRI image, and symptoms, five patients were considered to have leptomeningeal carcinomatosis (LMC) as well. Eighteen patients had other non-cranial organ metastases including bone, lung, pleura, liver, adrenal gland (Additional file 5: Table S5). Subsequent treatment received in this cohort were TKI $(9,43 \%)$, TKI and stereotactic radiosurgery of BM (3, $14 \%)$, TKI and resection of BM $(1,5 \%)$, TKI and bevacizumab (1,5\%), chemotherapy $(3,14 \%)$, whole brain irradiation and best support care $(2,10 \%)$, best support care $(2,10 \%)$ (Table 1 and Additional file 5: Table S5). A total of 15 tumor tissues, 19 CSFs, and 21 plasmas were sampled. Tumor tissues included primary tumors (5) and metastatic tumors from lymph node (7), lung (1), brain (1), pleura (1). Activating mutations were identified in tumor samples including EGFR L858R, EGFR T790M, EGFR exon 19 deletion, ERBB2 amplification, $A L K$ fusion, KRAS G12C.

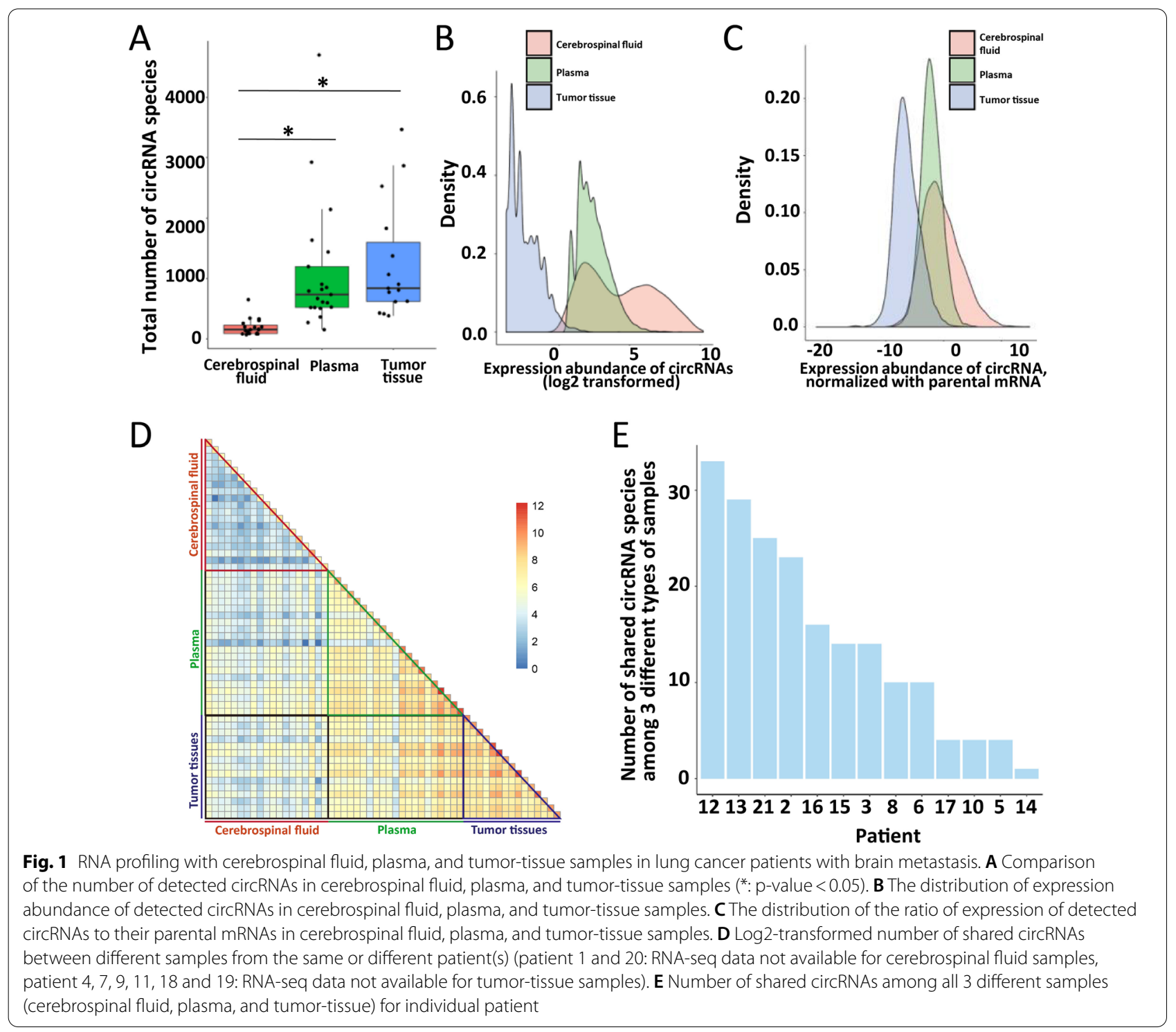




\section{The heterogeneity of CSF-circRNA in lung cancer}

Total rRNA depleted RNA was sequenced for profiling circRNA in CSF, plasma and tumor tissue. The total number of circRNA species detected in CSF was significantly less than in plasma and tumor samples $(p<0.05$, Fig. 1A), while the expression abundance of circRNAs in CSF was much higher (Fig. 1B and Additional file 6: Figure S2) even when normalized with parental mRNAs (Fig. 1C and Additional file 6: Figure S3). There was no significant correlation between expression abundance of circRNAs and their parental mRNAs among all the patients across sample types (Additional file 6: Figure S4). The number of commonly shared circRNA species among all three types of samples varied in patients (Fig. $1 \mathrm{E}$ and Additional file 6: Figure S5). Less shared circRNA species were found in CSF than in tumor tissue and plasma among patients (Fig. 1D), revealing high inter-patient heterogeneity of CSF. Within the individual patient, less shared circRNAs were found between tumor tissue and CSF than between tumor tissue and plasma (bottom, Fig. 1D), implying that circRNAs profile in CSF might bear more resemblance to that in brain metastatic tumors rather than in non-cranial tumors.

\section{Clinical characteristics and circRNA}

Total circRNA species and CSF-circRNAs abundance displayed a trend of increase along with lines of therapies in BM lung cancer patients $(p=0.26, p=0.66$ Additional file 6: Figure S6), indicating a potential association between CSF-circRNAs and clinical outcomes. CSF-circRNA expression had a significant negative correlation with the CSF-ctDNA concentration $(r=-0.76, p=0.028$, Additional file 6: Figure S7). The trend of negative correlation between circRNA and ctDNA was also found in tumor tissue and plasma but without significance (tumor tissue: $r=-0.34, p=0.23$; plasma: $r=-0.21, p=0.42$, Additional file 6: Figure S7). Clinical features including sex, smoking status, and genomic alterations such as EGFR/KRAS/TP53/ALK mutations were not associated with total counts or abundance of CSF-circRNAs (Additional file 6: Figure S8, CSF). In plasma, circRNA showed significantly higher expression abundance in mutant TP53 than in wild type. The total number of circRNA species but not expression abundance was higher in plasma from male patients and in tumor tissue from non-smokers and $A L K$ mutations carriers (Additional file 6: Figure S8, plasma and tissue) than in their according counterpart.

\section{Identification of CSF-circRNAs correlated with clinical outcome}

A total of 2,897 circRNAs were identified in CSF from $\mathrm{BM}$ lung cancer patients. The percentage of circRNAs was the same as previously found in brain tissue [44] and ranged from 47 to $81 \%$ of the total CSF-circRNAs in each patient (Additional file 5: Table S6). Some criteria were applied to effectively screen for cancer-related CSFcircRNAs (Additional file 6: Figure S9). Initially, 1036 circRNAs previously reported in human normal brain tissue [44] were excluded. To exclude possible normal tissue circRNAs, only those circRNAs, which appeared in tumor tissues at least two patients, were kept for further analysis. Finally, sixty potentially cancer-related circRNAs were remained (Fig. 2A). The expression abundance of 60 selected circRNAs was identified in individual patient (Fig. 2B).

The median OS of total group was 17.0 months with a median 34.1 months follow-up (Fig. 2C). Patients were divided into CSF circRNA-abundant and CSF circRNAdeficient groups based on the top 25\% (the cutoff is 7) of expressed circRNAs among 60 selected candidates. Patients in CSF circRNA-deficient group $(\leq 7$ of 60 selected circRNAs) had longer OS than CSF circRNAabundant ( $>7$ of 60 selected circRNAs) $(p=0.037$, in Fig. 2D). An insignificant positive correlation was observed between the number of detected candidates among 60 CSF-circRNAs and BM lesion size, which was the longest diameter of the largest lesion (Fig. 2E, Additional file 5: Table S5).

\section{Sixty CSF-circRNAs were associated with cell proliferation and differentiation pathways}

To study the biological functions of the sixty circRNAs, circRNA-miRNA-mRNA network (method) was constructed. Cancer-associated pathways enriched in target genes of the 60 CSF-circRNAs were analyzed by using DAVID (Fig. 3A). Majority of target genes were correlated with increased cell proliferation and differentiation and reduced apoptotic process. Both canonical [45] and noncanonical Wnt signaling [46] pathways, which have been known to support tumor metastasis [47,48], had the most enrichment of target genes (Fig. 3B, C). KEGG pathway enrichment analyses were performed using target genes of mRNA co-expressed with the sixty CSF-circRNAs and the transcriptional misregulation in cancer was identified as one of the most enriched biological processes (Fig. 3D).

\section{Validation of selected WNT signalling pathway associated circRNAs}

Five of 60 candidates CSF-circRNAs, which were hsa circ_0003011 (parental RFWD2), hsa_circ_0008604 (parental RHOT1), hsa_circ_0002360 (parental RUNX1), hsa_circ_0009117 (parental RERE), and hsa_circ_0002588 (parental RBMS2), were annotated with top-rank WNT signaling pathways in circRNA-miRNA-mRNA interacting network and were selected for further validation (Fig. 4A).

RT-PCR was performed to examine circRNA expression in CSF from another $10 \mathrm{BM}$ lung adenocarcinoma patients 


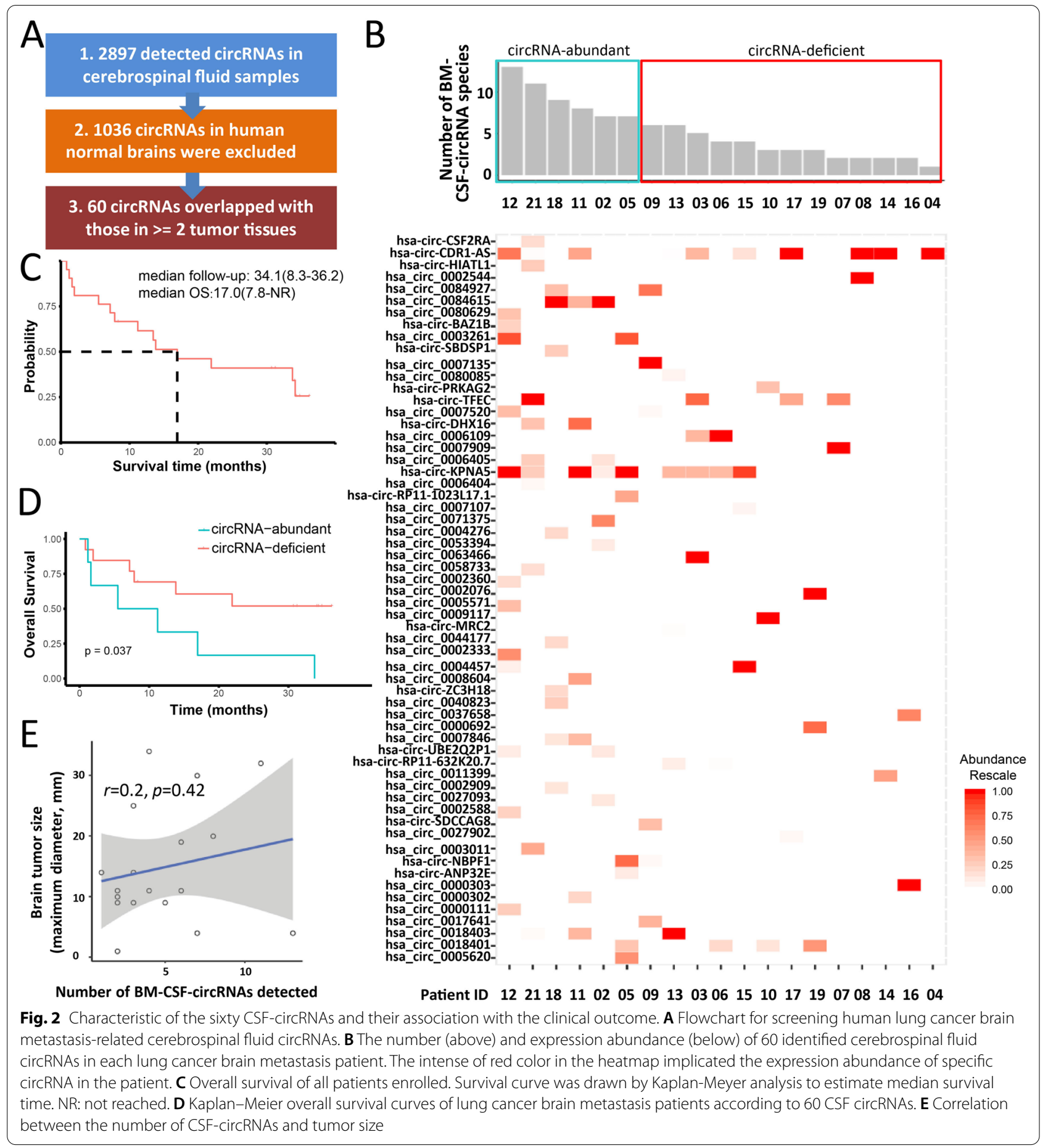

and two non-cancer patients as normal control. It was showed that circ_0002360, circ_0002588 and circ_0008604 were only identified in CSF from lung cancer patients (Fig. 4B) and NGS confirmed their sequences. Circ_0003011 and circ_0009117 were not found in CSF from either cancer or non-cancer patients.
Lentiviral shRNA vectors were constructed targeting the five circRNAs (hsa_circ_0003011, hsa_circ_0008604, hsa circ_0002360, hsa_circ_0009117, and hsa_circ_0002588) and A549 and HCC827 cell lines were infected. The levels of hsa_circ_0002360 and hsa_circ_0002588 significantly decreased with shRNA knock-down in HCC827 (Additional 
A

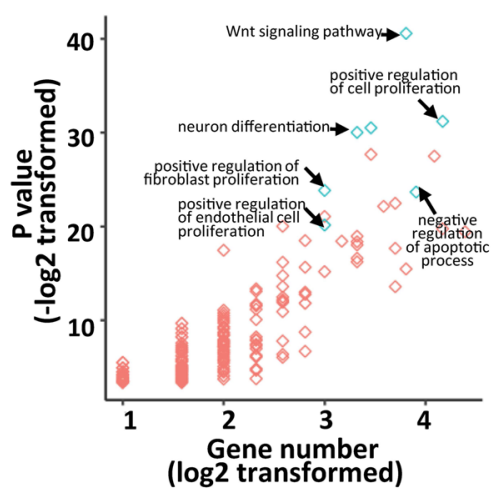

C

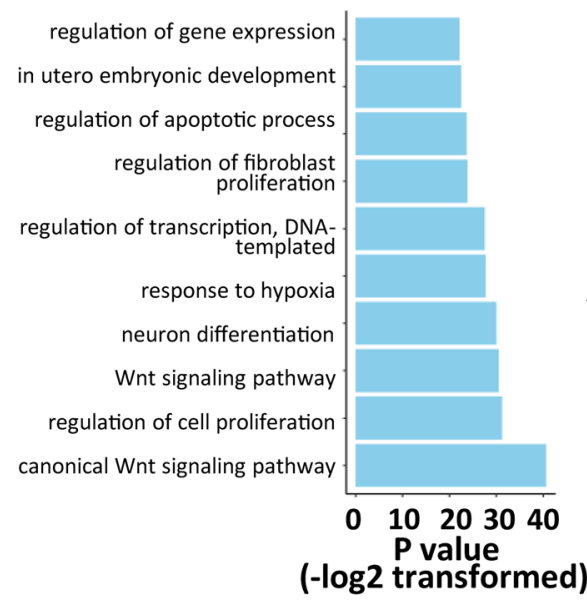

B

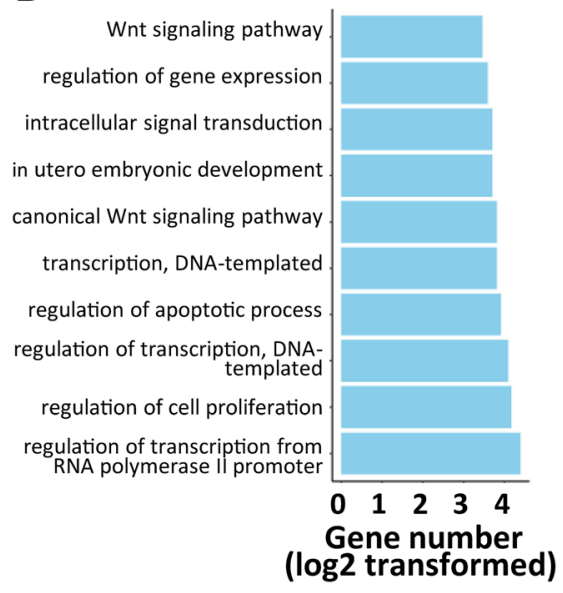

D

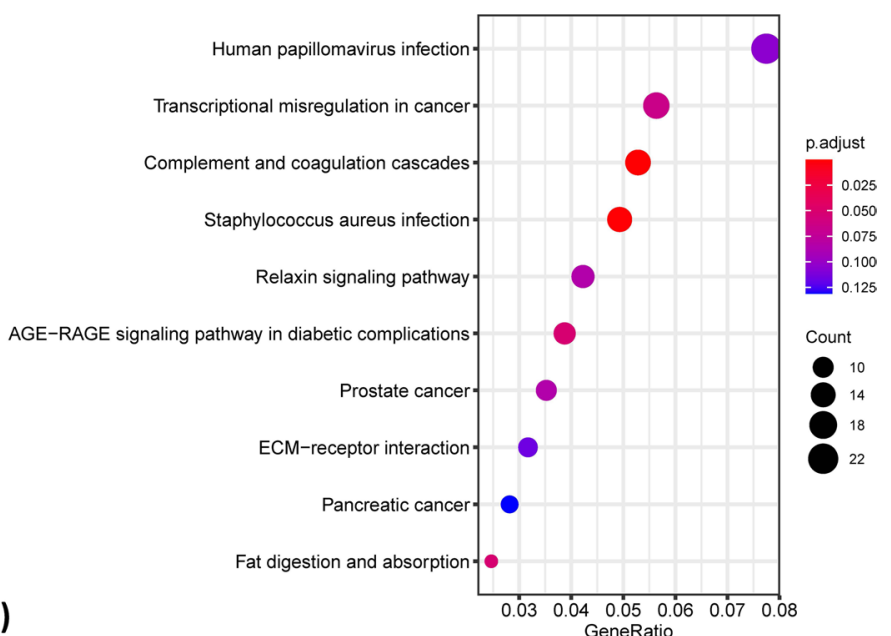

Fig. 3 Function annotations and enrichment for the target genes of 60 CSF circRNAs. A KEGG pathway enrichment analyses of the target genes of co-expressed mRNA of 60 CSF circRNAs. B Cancer-associated pathway using DAVID function annotation for the 60 CSF-circRNAs targeted genes. The scatter plots demonstrated all features with $\mathrm{p}$-value and gene counts. Positive regulation of cell proliferation and negative regulation of apoptosis were labelled. The detailed gene counts and p-value were shown in C, D. The vertical axis shows the annotated functions of the target genes. The horizontal axes show log2 transformed gene number and - log2 transformed p-value respectively. Only the most significantly enriched pathways were included

file 6: Figure S10A), and the proliferation of cells transfected with these shRNAs was greatly reduced (Fig. 4D). In A549 model, shRNAs significantly knocked down hsa circ_0003011, hsa_circ_0008604 and hsa_circ_0009117 (Additional file 6: Figure S10A) while exhibited negative impact on cell proliferation (Fig. 4D, Additional file 6: Figure S10B, D) and invasion ability of A549 cells was also undermined (Additional file 6: Figure S10C).

\section{Discussion}

Numerous reports have shown the potential roles of circRNAs in tumor metastasis [16, 18, 23, 24, 49, 50]. While most studies focused on tumor tissues, there are increasing interests in circRNAs analysis using liquid biopsies [50,51]. Given the inaccessibility of brain tumors from metastatic lung cancer patients, the combination of circRNA and liquid biopsy would provide more information to the treatment management. The frequently renewed cerebrospinal fluid keeps intimate contact with brain malignancies [8,9], may contain more tumor-specific molecules but fewer contaminations from blood cellular components than plasma [52], and thus may serve as a promising reservoir of BM-related circRNAs. However, little comparative analysis of circRNAs derive from tumor tissues, plasma and CSF has been reported in clinical settings. In this 


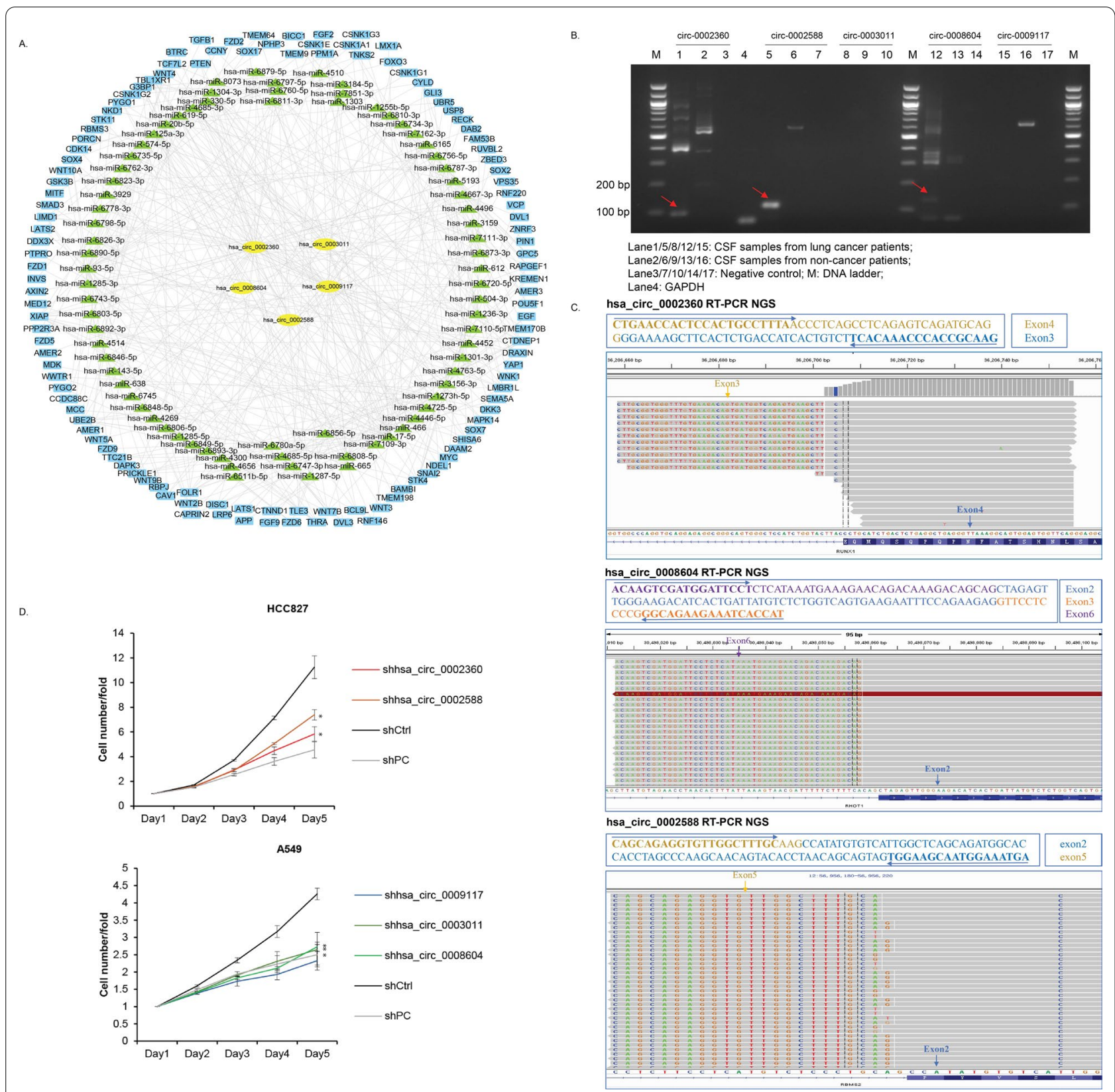

Fig. 4 Validation of potential roles of selected circRNAs. A circRNA-miRNA-mRNA network of the five WNT-associated CSF-circRNAs. B Quantification of five circRNAs by RT-PCR and agarose gel electrophoresis. GAPDH was used as internal control. C NGS of circRNAs after RT-PCR. D Detection of cell growth by Celigo automated imaging and cell counting platform. Triple experiments were performed. Values were represented as mean \pm SD. T-test was used for comparison. *: P $<0.05$

study, CSF-circRNAs gained more diversity compared to circRNAs from tumor and plasma across patients, indicating the inter-patient heterogeneity. A total of 60 potential prognosis-related CSF-circRNAs were identified and they are reasonably deemed to be BM-associated on the basis of fact that free circRNAs escaping from primary lung tumors to the brain metastases is strictly regulated by blood-brain barrier.

CircRNAs' function is known to be more associated with their target genes rather than parental genes. Our results showed that the candidate target genes of the 60 circRNAs were significantly enriched in the Wnt signaling 
pathways, which were tightly associated with cancer development [47], maintenance of cancer stem cells [53], and metastasis [47]. The presence of FZD2 and its ligand WNT5A (associated circRNAs: hsa_circ_0002360, hsa-circ-DHX16, hsa_circ_0002544, hsa_circ_0008604, hsa_circ_0009117) implied the enhanced epithelial to mesenchymal transition and cell migration [47]. In addition, MYC (associated circRNAs: hsa_circ_0002588, hsa_circ_0007909, hsa_circ_0008604), the well-known metastasis gene for lung cancer [54], was also identified. These data support the association between brain metastasis and the 60 circRNAs and guarantee future study on development and metastasis of lung cancer.

Five WNT-pathway related circRNAs including hsa circ_0002360 (parental RUNX1), hsa_circ_0002588 (parental RBMS2), hsa_circ_0009117 (parental RERE), hsa circ_0003011 (parental RFWD2), and hsa_circ_0008604 (parental RHOT1) were demonstrated to function in in vitro assays. As previously reported, hsa_circ_0002360 (parental RUNX1) overexpressed in lung adenocarcinoma and hsa_circ_000-2360/hsa-mir-3620-5p/PHF19 might interact in the progression of lung adenocarcinoma under cicrRNA-miRNA-mRNA networks [22]. Our results also showed that hsa_circ_0002360 (parental RUNX1) as one of 60 candidates circRNAs shared between CSF and tumor samples might function in the lung adenocarcinoma cell proliferation, migration and invasion.

Among the target genes of 5 validated circRNAs in Wnt pathway, many were identified to function in progression and metastasis of lung cancer. Wnt signaling is of great importance and it is common in lung cancer to have overexpression of Wnt-1/2/3/5a and other components (e.g. FZD, DVL, PORCN, TCF) $[55,56]$, which were predicted as the target genes for hsa_circ_0002360 (target genes: Wnt-2/3/5a/ FZD/ DVL1/PORCN/TCF), hsa_circ_0003011 (target genes: FZD), hsa_circ_0008604 (target genes: Wnt-3/5a), hsa_circ_0009117 (target genes: Wnt-2/3/5a/FZD/ DVL3/PORCN/TCF). In lung cancer, DVLs was reportedly to activate Wnt pathway [57] and brain metastasis had increased expression of DVL1 and DVL3 [58], which are potential targets of hsa_circ_0002360 and hsa_circ_0009117, respectively.

There were some limitations in this study. The validation experiments were preliminary and the function of these circRNAs were not comprehensively demonstrated. Due to the small size of group of patients, the survival analysis results of circRNAs are not convincing enough. However, further studies are guaranteed on the basis of our study.

Collectively, this study provided a leading and fundamental circRNAs profiles of lung cancer brain metastasis by using CSF.

\section{Conclusions}

Our data showed that the 60 circRNAs might play important roles in regulating biological functions and cancer-related signaling pathways and might be associated with cell proliferation and invasion process. CSFcircRNAs might be promising biomarkers in the lung cancer patients with brain metastases.

\section{Abbreviations \\ circRNAs: Circular RNAs; ncRNAs: Noncoding RNAs; BM: Brain metastasis; CSF: Cerebrospinal fluid; ADC: Adenocarcinoma; TMM: Trimmed means of M-values: DAVID: Database for annotation, visualization and integrated discovery; PC: Positive control; PBS: Phosphate-buffered saline; ctDNA: Circulating tumor DNA; OS: Overall survival; HR: Hazard ratio; PFS: Progression-free survival.}

\section{Supplementary Information}

The online version contains supplementary material available at https://doi. org/10.1186/s12967-022-03274-1.

Additional file 1: Table S1. QC information.

Additional file 2: Table S2. FPKM of all samples.

Additional file 3: Table S3. circRNA-miRNA-mRNA.

Additional file 4: Table S4. circRNA-coexpressed mRNA symbol.

Additional file 5: Table S5. Clinical data pertaining to brain/leptomeningeal metastasis. Table S6. CSF circRNAs in this study and normal brain circRNAs previously reported.

Additional file 6. Additional figures S1 to S10.

\section{Acknowledgements}

We would like to thank the patients and their families, as well as the investigators and research staff involved.

\section{Authors' contributions}

ZW, YLW designed this study. ZW, ANL, HYT, QZ performed the data acquisition. RC, HB, XW, ZW, XC performed data analysis. RC, RYY, ZW, QXO wrote the manuscript. YLW, ZW supervised the present study. All authors read and approved the final manuscript.

\section{Funding}

This work was supported by Guangzhou Science and Technology Project (grant 201804010477 to Zhen Wang), CSCO-Roche Research Fund (grant Y-2019Roche-022 to Zhen Wang), CSCO Research Fund (Y-2019AZMS-0034 to Zhen Wang), Public Interest by National Health and Family Control Committee (grant 201402031 to Yi-Long Wu) and Guangdong Provincial People's Hospital Research Fund.

\section{Availability of data and materials}

The data used and/or analyzed during the current study are available from the corresponding author on reasonable request.

\section{Declarations}

\section{Ethics approval and consent to participate}

The study was approved by the Ethical Committee of the Guangdong Provincial People's Hospital (No. GDREC2020188H). Written consent was obtained from each patient.

\section{Consent for publication}

Informed consent form was obtained from each patient. 


\section{Competing interests}

Ran Cao, Ruoying Yu, Xiaoxi Chen, Hua Bao, Qiuxiang Ou and Xue Wu are the shareholders or employees of Nanjing Geneseeq Technology Inc. The remaining authors have no competing interest to declare.

\section{Author details}

${ }^{1}$ Guangdong Provincial People's Hospital, Guangdong Academy of Medical Sciences, Guangdong Lung Cancer Institute, Zhong Shan 2nd road 106, Guangzhou 510080, Guangdong, China. ${ }^{2}$ Geneseeq Research Institute, Nanjing Geneseeq Technology Inc., Nanjing, Jiangsu, China. ${ }^{3}$ Guangdong Cardiovascular Institute, Guangzhou, China.

\section{Received: 17 August 2021 Accepted: 24 January 2022}

\section{Published online: 05 February 2022}

\section{References}

1. Yamanaka R. Medical management of brain metastases from lung cancer (Review). Oncol Rep. 2009;22:1269-76.

2. Delattre JY, Krol G, Thaler HT, Posner JB. Distribution of brain metastases. Arch Neurol. 1988;45:741-4.

3. Waqar SN, Waqar SH, Trinkaus K, Gadea CA, Robinson CG, Bradley J, Watson MA, Puri V, Govindan R, Morgensztern D. Brain metastases at presentation in patients with non-small cell lung cancer. Am J Clin Oncol. 2018:41:36-40.

4. Patel RR, Mehta MP. Targeted therapy for brain metastases: improving the therapeutic ratio. Clin Cancer Res. 2007;13:1675-83.

5. Seoane J, De Mattos-Arruda L, Le Rhun E, Bardelli A, Weller M. Cerebrospinal fluid cell-free tumour DNA as a liquid biopsy for primary brain tumours and central nervous system metastases. Ann Oncol. 2019;30:211-8.

6. Siravegna G, Marsoni S, Siena S, Bardelli A. Integrating liquid biopsies into the management of cancer. Nat Rev Clin Oncol. 2017;14:531-48.

7. De Mattos-Arruda L, Mayor R, Ng CKY, Weigelt B, Martinez-Ricarte F, Torrejon D, Oliveira M, Arias A, Raventos C, Tang J, et al. Cerebrospinal fluid-derived circulating tumour DNA better represents the genomic alterations of brain tumours than plasma. Nat Commun. 2015;6:8839.

8. Redzic Z. Molecular biology of the blood-brain and the blood-cerebrospinal fluid barriers: similarities and differences. Fluids Barriers CNS. 2011:8:3.

9. Ghersi-Egea JF, Strazielle N, Catala M, Silva-Vargas V, Doetsch F, Engelhardt B. Molecular anatomy and functions of the choroidal blood-cerebrospinal fluid barrier in health and disease. Acta Neuropathol. 2018;135:337-61.

10. Shen F, Zhang Y, Yao Y, Hua W, Zhang HS, Wu JS, Zhong P, Zhou LF. Proteomic analysis of cerebrospinal fluid: toward the identification of biomarkers for gliomas. Neurosurg Rev. 2014;37:367-80.

11. Xi X, Li T, Huang Y, Sun J, Zhu Y, Yang Y, Lu ZJ. RNA biomarkers: frontier of precision medicine for cancer. Noncoding RNA. 2017;3:1-9.

12. Shang Q, Yang Z, Jia R, Ge S. The novel roles of circRNAs in human cancer. Mol Cancer. 2019;18:6.

13. Zhu Q, Lu G, Luo Z, Gui F, Wu J, Zhang D, Ni Y. CircRNA circ_0067934 promotes tumor growth and metastasis in hepatocellular carcinoma through regulation of miR-1324/FZD5/Wnt/ß-catenin axis. Biochem Biophys Res Commun. 2018;497:626-32.

14. Zong L, Sun Q, Zhang H, Chen Z, Deng Y, Li D, Zhang L. Increased expression of circRNA_102231 in lung cancer and its clinical significance. Biomed Pharmacother. 2018:102:639-44.

15. Rong D, Lu C, Zhang B, Fu K, Zhao S, Tang W, Cao H. CircPSMC3 suppresses the proliferation and metastasis of gastric cancer by acting as a competitive endogenous RNA through sponging miR-296-5p. Mol Cancer. 2019;18:25

16. Hsiao K-Y, Lin Y-C, Gupta SK, Chang N, Yen L, Sun HS, Tsai S-J. Noncoding effects of circular RNA CCDC66 promote colon cancer growth and metastasis. Can Res. 2017:77:2339.

17. Gao D, Qi X, Zhang X, Fang K, Guo Z, Li L. hsa_circRNA_0006528 as a competing endogenous RNA promotes human breast cancer progression by sponging miR-7-5p and activating the MAPK/ERK signaling pathway. Mol Carcinog. 2019:58:554-64.

18. Shen B, Wang Z, Li Z, Song H, Ding X. Circular RNAs: an emerging landscape in tumor metastasis. Am J Cancer Res. 2019:9:630-43.
19. Sekar S, Liang WS. Circular RNA expression and function in the brain. Non-coding RNA Res. 2019;4:23-9.

20. Rybak-Wolf A, Stottmeister C, Glazar P, Jens M, Pino N, Giusti S, Hanan M, Behm M, Bartok O, Ashwal-Fluss R, et al. Circular RNAs in the mammalian brain are highly abundant, conserved, and dynamically expressed. Mol Cell. 2015;58:870-85.

21. Zhang Z, Yang T, Xiao J. Circular RNAs: promising biomarkers for human diseases. EBioMedicine. 2018;34:267-74.

22. Yan $Y$, Zhang $R$, Zhang $X$, Zhang A, Zhang Y, Bu X. RNA-Seq profiling of circular RNAs and potential function of hsa_circ_0002360 in human lung adenocarcinom. Am J Transl Res. 2019;11:160-75.

23. Hu Z-Q, Zhou S-L, Li J, Zhou Z-J, Wang P-C, Xin H-Y, Mao L, Luo C-B, Yu S-Y, Huang $X-W$, et al. Circular RNA sequencing identifies CircASAP1 as a key regulator in hepatocellular carcinoma metastasis. Hepatology. 2020;72:906-22.

24. Yu J, Xu Q-g, Wang Z-g, Yang Y, Zhang L, Ma J-z, Sun S-h, Yang F, Zhou W-p. Circular RNA cSMARCA5 inhibits growth and metastasis in hepatocellular carcinoma. J Hepatol. 2018;68:1214-27.

25. Chen L, Nan A, Zhang N, Jia Y, Li X, Ling Y, Dai J, Zhang S, Yang Q, Yi Y, Jiang Y. Circular RNA 100146 functions as an oncogene through direct binding to miR-361-3p and miR-615-5p in non-small cell lung cancer. Mol Cancer. 2019:18:13.

26. Maass PG, Glažar P, Memczak S, Dittmar G, Hollfinger I, Schreyer L, Sauer AV, Toka O, Aiuti A, Luft FC, Rajewsky N. A map of human circular RNAs in clinically relevant tissues. J Mol Med. 2017;95:1179-89.

27. Wang X, Gao Y, Shan C, Lai M, He H, Bai B, Ping L, Rong Q, Ai R, Wen L, et al Association of circulating tumor DNA from the cerebrospinal fluid with high-risk CNS involvement in patients with diffuse large B-cell lymphoma. Clin Transl Med. 2021;11:e236.

28. Yang Z, Yang N, Ou Q, Xiang Y, Jiang T, Wu X, Bao H, Tong X, Wang X, Shao $Y W$, et al. Investigating novel resistance mechanisms to third-generation EGFR tyrosine kinase inhibitor osimertinib in non-small cell lung cancer patients. Clin Cancer Res. 2018;24:3097-107.

29. Bolger AM, Lohse M, Usadel B. Trimmomatic: a flexible trimmer for Illumina sequence data. Bioinformatics. 2014;30:2114-20.

30. Koboldt DC, Zhang Q, Larson DE, Shen D, McLellan MD, Lin L, Miller CA Mardis ER, Ding L, Wilson RK. VarScan 2: somatic mutation and copy number alteration discovery in cancer by exome sequencing. Genome Res. 2012;22:568-76.

31. Wang K, Li M, Hakonarson H. ANNOVAR: functional annotation of genetic variants from high-throughput sequencing data. Nucleic Acids Res. 2010;38:e164.

32. Newman AM, Bratman SV, Stehr H, Lee LJ, Liu CL, Diehn M, Alizadeh AA. FACTERA: a practical method for the discovery of genomic rearrangements at breakpoint resolution. Bioinformatics. 2014;30:3390-3.

33. Chen S, Huang V, Xu X, Livingstone J, Soares F, Jeon J, Zeng Y, Hua JT, Petricca J, Guo H, et al. Widespread and functional RNA circularization in localized prostate cancer. Cell 2019;176:831-843 e822.

34. Lawrence M, Huber W, Pages H, Aboyoun P, Carlson M, Gentleman R, Morgan MT, Carey VJ. Software for computing and annotating genomic ranges. PLoS Comput Biol. 2013:9:e1003118.

35. Van Der Steen N, Lyu Y, Hitzler AK, Becker AC, Seiler J, Diederichs S. The circular RNA landscape of non-small cell lung cancer cells. Cancers (Basel). 2020;12:1091.

36. Vo JN, Cieslik M, Zhang Y, Shukla S, Xiao L, Zhang Y, Wu YM, Dhanasekaran SM, Engelke CG, Cao X, et al. The landscape of circular RNA in cancer. Cell 2019:176:869-881 e813.

37. Hansen TB, Jensen TI, Clausen BH, Bramsen JB, Finsen B, Damgaard CK, Kjems J. Natural RNA circles function as efficient microRNA sponges. Nature. 2013:495:384-8.

38. Enright A, John B, Gaul U, TuschI T, Sander C, Marks D. MicroRNA targets in Drosophila. Genome Biol. 2003;4:1-27.

39. Lin YC, Lee YC, Chang KL, Hsiao KY. Analysis of common targets for circular RNAs. BMC Bioinformatics. 2019;20:372.

40. Dweep H, Sticht C, Pandey P, Gretz N. miRWalk-database: prediction of possible miRNA binding sites by "walking" the genes of three genomes. J Biomed Inform. 2011;44:839-47.

41. Shannon P, Markiel A, Ozier O, Baliga NS, Wang JT, Ramage D, Amin N, Schwikowski B, Ideker T. Cytoscape: a software environment for integrated models of biomolecular interaction networks. Genome Res. 2003:13:2498-504. 
42. da Huang W, Sherman BT, Lempicki RA. Systematic and integrative analysis of large gene lists using DAVID bioinformatics resources. Nat Protoc. 2009:4:44-57.

43. Sun R, Wu J, Chen Y, Lu M, Zhang S, Lu D, Li Y. Down regulation of Thrombospondin2 predicts poor prognosis in patients with gastric cancer. Mol Cancer. 2014;13:225.

44. Enuka Y, Lauriola M, Feldman ME, Sas-Chen A, Ulitsky I, Yarden Y. Circular RNAs are long-lived and display only minimal early alterations in response to a growth factor. Nucleic Acids Res. 2016:44:1370-83.

45. Gordon MD, Nusse R. Wnt signaling: multiple pathways, multiple receptors, and multiple transcription factors. J Biol Chem. 2006;281:22429-33.

46. Kohn AD, Moon RT. Wnt and calcium signaling: beta-catenin-independent pathways. Cell Calcium. 2005;38:439-46.

47. Zhan T, Rindtorff N, Boutros M. Wnt signaling in cancer. Oncogene. 2017;36:1461-73.

48. Jin $\mathrm{K}$, Li T, van Dam H, Zhou F, Zhang L. Molecular insights into tumour metastasis: tracing the dominant events. J Pathol. 2017;241:567-77.

49. Xu H, Wang C, Song H, Xu Y, Ji G. RNA-Seq profiling of circular RNAs in human colorectal Cancer liver metastasis and the potential biomarkers. Mol Cancer. 2019;18:8-8.

50. He F, Zhong X, Lin Z, Lin J, Qiu M, Li X, Hu Z. Plasma exo-hsa_cirCRNA_0056616: A potential biomarker for lymph node metastasis in lung adenocarcinoma. J Cancer. 2020;11:4037-46.

51. de Fraipont F, Gazzeri S, Cho WC, Eymin B. Circular RNAs and RNA splice variants as biomarkers for prognosis and therapeutic response in the liquid biopsies of lung cancer patients. Front Genet. 2019;10:390.

52. Kopkova A, Sana J, Machackova T, Vecera M, Radova L, Trachtova K, Vybihal V, Smrcka M, Kazda T, Slaby O, Fadrus P. Cerebrospinal fluid MicroRNA signatures as diagnostic biomarkers in brain tumors. Cancers. 2019;11:1546.

53. Reya T, Clevers H. Wnt signalling in stem cells and cancer. Nature. 2005;434:843-50

54. Rapp UR, Korn C, Ceteci F, Karreman C, Luetkenhaus K, Serafin V, Zanucco E, Castro I, Potapenko T. MYC is a metastasis gene for non-small-cell lung cancer. PLoS ONE. 2009;4:e6029.

55. Stewart DJ. Wnt signaling pathway in non-small cell lung cancer. JNCl. 2014. https://doi.org/10.1093/jnci/djt356.

56. Tennis M, Van Scoyk M, Winn RA. Role of the Wnt signaling pathway and lung cancer. J Thorac Oncol. 2007;2:889-92.

57. Mazieres J, He B, You L, Xu Z, Jablons DM. Wnt signaling in lung cancer. Cancer Lett. 2005;222:1-10.

58. Kafka A, Tomas D, Beroš V, Pećina HI, Zeljko M, Pećina-Šlaus N. Brain metastases from lung cancer show increased expression of DVL1, DVL3 and beta-catenin and down-regulation of E-cadherin. Int J Mol Sci. 2014;15:10635-51.

\section{Publisher's Note}

Springer Nature remains neutral with regard to jurisdictional claims in published maps and institutional affiliations.

Ready to submit your research? Choose BMC and benefit from:

- fast, convenient online submission

- thorough peer review by experienced researchers in your field

- rapid publication on acceptance

- support for research data, including large and complex data types

- gold Open Access which fosters wider collaboration and increased citations

- maximum visibility for your research: over 100M website views per year

At BMC, research is always in progress.

Learn more biomedcentral.com/submissions 